\title{
The Design of Fast Storing Testing System Based on Projectile-borne
}

\author{
Tang Yufa, Zhang He ,Chen Yan \\ ZNDY of Ministerial Key Laboratory, \\ Nanjing University of science \& technology of China \\ Nanjing, China
}

\begin{abstract}
A high-speed storage testing system based on C8051F340 is introduced in the paper. The system is small in size, with high impact and more hostile environment resistance, low power consumption. It can be placed in the projectile with diameter of $25 \mathrm{~mm}$ or above. Using the secondary memory technique, it can record relevant motion parameters of the projectile in flight in the real time very rapidly, with a similar function of aircraft "black box". What's more, the system has the advantage of holding the data when the power is removed. The data can be recovered via USB interface when retrieved to ensure the accuracy of the test.
\end{abstract}

Keywords-Fast storing, C8051F, Secondary memory, USB, Ferroelectric

\section{INTRODUCTION}

Fast memory technique, which has a wide application prospect, is an effective means to complete the parameter testing of operating condition for moving body in a special working environment [1]. However, the category of memory systems existing is few . And most of them don't have a high performance-to-price ratio to meet the author's requirements of test environment. As we studied, the projectile's relevant testing parameters during movement have the following features: longer system testing time, higher test frequency, requirements of testing system fast storing data which is sent by peripheral circuit or device at the frequency of 2 $\mathrm{KHz}$, and the records of the time should not be less than 35 s.

This requires that the memory system should receive and store the data in real time, quickly and accurately. A long memory time is expected to ensure that the system can get the right data through USB interface quickly after being retrieved. As the system works in the projectile, a testing system in small size is required, and it's able to withstand the impact of the $5000 \mathrm{G}$ acceleration as well. Therefore a testing system with a low power consumption, high impact resistance must be designed according to author's research object. The following job is the development and designing of the system's hardware and software according to the requirements mentioned above.

\section{HARDWARE DESIGN OF THE FAST STORAGE SYSTEM}

\section{A. The selection of main components}

The memory testing system uses the C8051F340 from Silicon company as the core of MCU[2]. C8051F340 is a fully integrated mixed signal System-on-a-Chip MCU with a high-speed pipelined 8051-compatible microcontroller core(up to 48 MIPS). It has up to $64 \mathrm{kB}$ of on-chip Flash memory, up to 4352 Bytes of on-chip RAM(256+4 kB), a universal Serial Bus(USB) function controller with eight flexible endpoint pipes, an integrated trans-ceiver, and $1 \mathrm{kB}$ FIFO RAM. The system is also provided with four generalpurpose 16-bit timers, a true 10-bit 200 ksps differential/single-ended ADC with analog multiplexer. In addition, this MCU supports on-chip voltage reference and temperature sensor, supplied with two on-chip voltage comparators, a programmable counter/timer array(PCA)with five capture/compare modules and watchdog timer function, a on-chip Power-On Reset, a VDD monitor, and a missing clock detector. It incorpates up to 40 Port $\mathrm{I} / \mathrm{O}(5 \mathrm{~V}$ tolerant), SMBus/I2C, up to 2 UARTs, and enhanced SPI serial interfaces implemented in hardware, provided with a precision internal calibrated $12 \mathrm{MHz}$ internal oscillator and $4 \mathrm{x}$ clock multiplier as well. The C8051F340 operational power supply voltage range is 2.7 to $5.25 \mathrm{~V}$. The core of MCU can work in normal mode, idle mode ,or power-down mode, presenting a flexible solution of the power management which is suitable to the low-consuption application platform. And it is available in 48-pin TQFP,32pin LQFP, or 32-pin QFN packages.

As we learned from the parameters above, C8051F340 has up to $64 \mathrm{kB}$ of on-chip Flash memory with a finite capacity. If a huge universe of data storage is needed, we should have an external data memory, that's what the design of the system introduced. On account of a high storage frequency, a large amount, and a long storage time of testing data, it's important to select a suitable external memory in the testing system of projectile's parameter storage. For the present, SRAM, FLASH, NVRAM or FRAM is widely used in the memory system to store testing data at home and abroad[3]. SRAM requires the system always on electricity with a great power consumption, which is against the low power consumption and data will be lost easily when out of power. The system should be in the working state all the time to ensure the data remained; The most distinguished feature of the FLASH memory is that the data won't lost when power failure occurs, but its rewriting cycle is generally more than $5 \mathrm{~ms}$, even the fastest one also needs 200 us. So to the data test system with high frequency and large amount, data memory speed can't meet the requirements of real-time and high speed; Despite that NVRAM has the advantages of fast read/write speed as 
SRAM, remaining data when out of power as FLASH, it is not easy to make the lager size smaller for the use of thick model circuits. FRAM(ferroelectric memory), which do not need to refresh at times, performs write/read operations at bus speed with data memory capacity in power failure case. It offers excellent features such as an unlimited time to rewritie(more than 1016 times), low power consumption, and a strong anti-interference ability of electromagnetic.

According to the system's requirements of fast memory and data remained in power failure case, a $\mathrm{FM} 25 \mathrm{H} 20$ memory chip from the ramtron company is selected. The FRAM chip is serial-nonvolatile, supplied by a working voltage range of $2.7-3.6 \mathrm{~V}$, packaged in 8-pin TDFN, provided with a shape size of $5 \mathrm{~mm} \times 6 \mathrm{~mm} \times 0.75 \mathrm{~mm}$, a memory structure of $256 \mathrm{~KB} \times 8$, a bus write/read speed at $40 \mathrm{MHz}$, infinite read/write times, low current operation and a reliable data retention for 10 years. Moreover, with the industrial standard SPI (Serial Peripheral Interface) interface, FRAM is optimized in fast write/read capability and expansibility. The system chooses 4 chips of FM25H20, making the capacity meet the system's requirements.

Therefore, the combination of C8051F340 and FM25H20 can meet the functional requirement of fast memory.

\section{B. Design of Hardware Circuit}

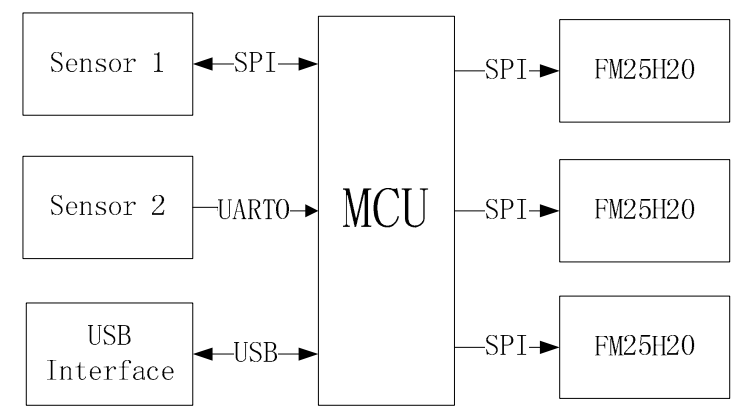

Figure 1. Principle block diagram

As shown in Figure.1, fast memory system consists of three sensor modules, a micro controller $\mathrm{MCU}$, four data memory FM25H20s and a USB interface. To reduce the size of the system to the extent, dual panel design is necessary in PCB production. There is only one USB interface for human-computer interaction, to read or playback the needed data more quickly after being retrieved, and to reduce board space of hardware as well.

The SPI interface in Single-Chip Microcomputer takes the operation of writing/reading to/from the sensor 1 and four $\mathrm{FM} 25 \mathrm{H} 20 \mathrm{~s}$ by the chip selection signal through the way of single master and several slaves mode.The sensor 2 and sensor 3 module communicate with MCU through UART0 and UART1 .

As C8051F340 has $4 \mathrm{~KB}$ XRAM, in order to ensure the integrity of the received data, the author adopts some methods as follows: firstly, MCU stores the data from sensors in the XRAM after proper data processing; secondly, put the group of data in XRAM into the memory FM25H20 after completing receiving a group of data, which is also called the secondary storage technology. The usage of secondary storage makes it faster to save data in case of data being lost during chip selection.

The system stores the data from sensors in FM25H20, and will be connected via USB to communicate with PC after being retrieved. Further processing and analysis are made on PC to get the flight state during projectile's movement.

As there are other equipments working on the projectile, the power supply can be obtained from the main power via a voltage conversion process which converts into a system voltage of $3.3 \mathrm{~V}$. Considering the limited volume and space of the tested object, a lot of work in the system's miniaturization has been done. Small size, low power consumption, fast storing speed are the highlight of the system: The system's circuit module can be packaged in a cylinder sized at $25 \mathrm{~mm}$ diameter, $25 \mathrm{~mm}$ length;And it works at a power consumption of $40 \mathrm{~mA}$, while the static power consumption is lower than $5 \mathrm{~mA}$.

\section{SOFTWARE DESIGN OF THE FAST STORAGE SYSTEM}

\section{A. Software design of storage module}

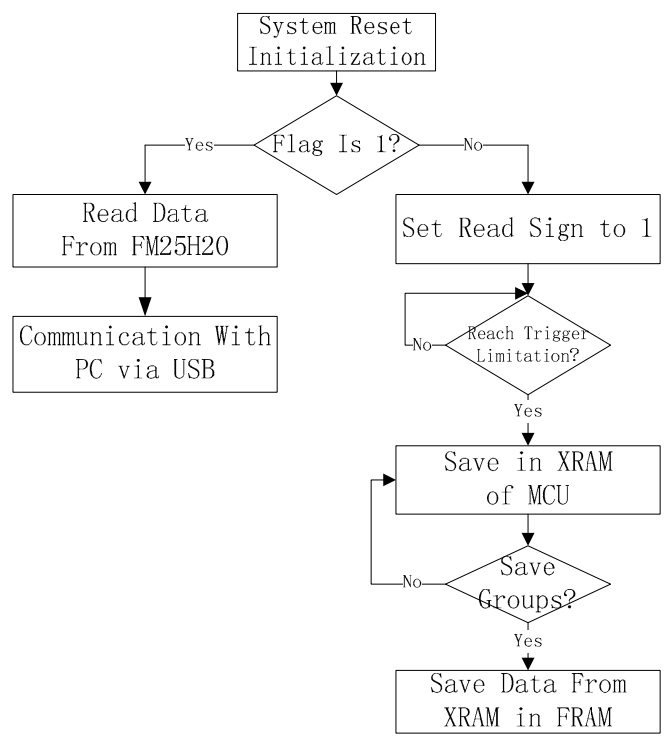

Figure 2. Software flowchart of fast-storage system

Since a lot of prepared work, such as sealing process, has to be done after the measured object being powered up, about 10 minutes is needed normally. During this period, the flag of write/read is initialized to zero, for the system is powered up first time. Meanwhile, only the program of limit detection is opened, and the function of storing is closed to save electric energy. As shown in Figure.3, then the projectile flies out and reaches the limit velocity, storage module begins to work at full speed, storing the received data in the XRAM of MCU. After finishing receiving a 
group of data, system invokes FRAM storing subprogram until the end of flight.

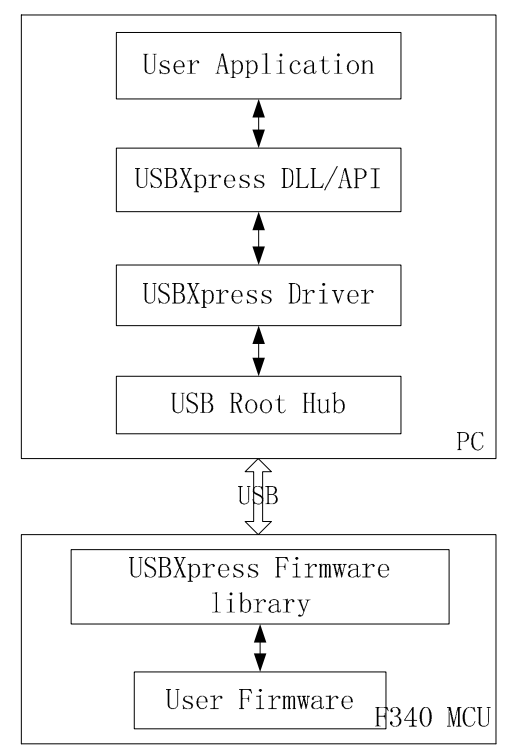

Figure 3. USBXpress Data Flow

When the projectile has been recovered, the system is powered on again. This time the read/write flag turns out to be 1 . Then the system invokes the FRAM reading subprogram, and communicates with PC via USB communication module in fast storage system. Data processing is done by MATLAB in PC, and the results of the test can be analyzed.

\section{B. Software design of communication module}

The software design of communication module adopts a USB Xpress software setups offered by Silicon Laboratories. It is designed for the USB interface of devices such as C8051F340, providing a complete software solution between PC and lower level computer, greatly simplifying the development work of the USB communication application and relevant driver. Figure. 4 shows the data flowchart of USB Xpress[4].

1) The program of lower level computer

Under the compile environment of Keil uVision3, the USB communication of equipment can be designed using the API functions in USBXpress tool kit, after adding the C8051F340.h、USB_API.h and USBX_F34X.LIB folders to the project[5]. And USB clock and USB interface should be initialized before data transmission, the functions involved concludes:
USB_Clock_Start ( )
USB_Init( )
Block_Write( )

$$
\begin{aligned}
& / * \text { initialize USB clock*/ } \\
& / * \text { enable USB interface*/ } \\
& / * \text { writing data to PC via } \\
& / * \text { reading data from PC via }
\end{aligned}
$$

$\mathrm{USB} * /$

$\mathrm{USB} * /$
Get_Interrupt_Source() /*getting the source of triggering API interrupt*/

USB Int Enable()

USB_Int_Disable()

USB_Disable()

USB_Suspend()

USB Get Library Version()/*return USB Xpress Library Version */

The function of API is achieved in the interrupt, thus an interrupt vector is needed. Among them, 0x008B is the interrupt vector of $\mathrm{F} 34 \mathrm{X}$ equipment. In the main function the program can't enter interrupts only when the USB interrupt(by invoking USB_Int_Enable function) is allowed. The interrupt flag should be gotten by the function of Get_Interrupt_Source once in the Interrupt Service Routine, and relevant operation can be executed later.

2) Client Application Program

It is important that SiUSBXp.dll 、SiUSBXp.h and SiUSBXp.lib folders should be added in advance when developing client USB application program under the $\mathrm{VC}++6.0$ compile environment. And before running the client application program, drivers of PC should be set up in order to make PC recognize USB equipment and communicate with it.

\section{DYNAMIC TEST AND DATA PROCESSING}

The following curve illustrated in Figure.5-7, is achieved after applying the fast storage system to the relevant parameters test of measurand.

Figure. 5 shows the dynamic testing ground, in which is a non-magnetic rotation table to simulate the circumferential direction motion situation of projectile; Figure. 6 shows the circumferential moving situation when it's with a elevation angle. The figure is obtained by subsequence processing with the data which has been stored via USB interface after the system being retrieved. It reflects the laws of acceleration in the fast storage system, which is in a circumferential direction motion.

Figure.7 shows that magnetic sensors are keeping cutting the geomagnetic lines to generate a periodic change of signal. A periodic change shows a revolution of the fast storage system .It comes to a conclusion from the figures that this system can measure the attitude information in any moments.

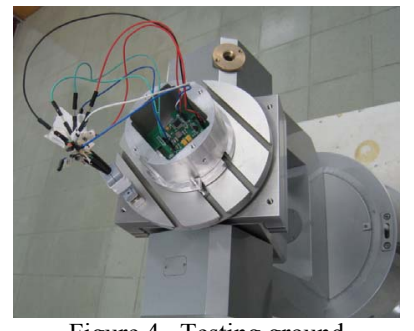

Figure 4. Testing ground

(1)

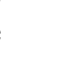

.




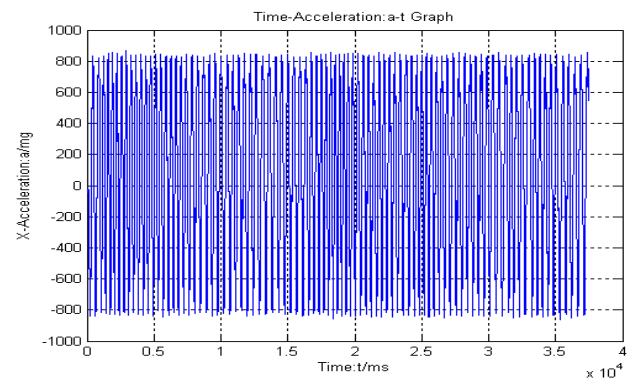

Figure 5. Circumferential acceleration varying curve over time

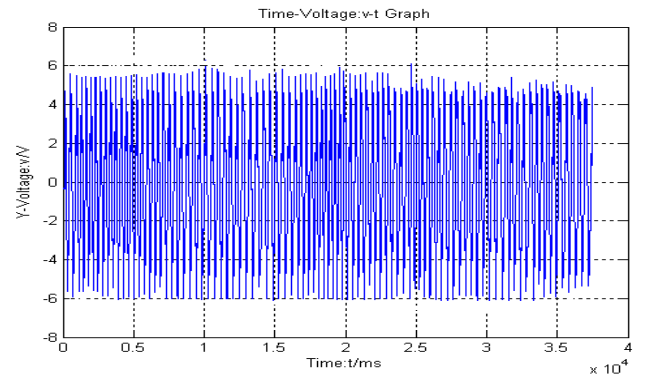

expounded 。 Experiments are made to testify the correctness of data storage in earlier stage and data processing in the later stage as well. Besides, the system, which is small in size, also has a high speed and a large capacity to store the collecting in low power . Therefore it's suitable for mini -sized measured objects in many applications which require low consumption. The USB interface improve the system's portability and convenience, providing a feasible scheme to develop an engineering.

\section{REFERENCES}

[1] Jiang Xiaohua,Li Haojie,Zhang He. Research on Micro-stored in-shell Data Acquisition System. Journal of Ballistics[J],2002,PP:57-61.

[2] Silicon Laboratories.C8051F34x User's Manual.

[3] Jiang Xiaohua. The Research of Distance measurement Technique on Turns Counting for Small Caliber Fuze. Nanjing University of Science \& Technology[D],2003,PP:76-79.

[4] Silicon Laboratories.AN169 USBXpress Programmer's Guide.

[5] Li Tongfei. The Design of USB Communication Based on USBXpress Development Package of C8051F340. Microcontrollers \& Embedded Systems[J],2010,PP:74-75.

Figure 7. Circumferential attitude angle varying curve over time

\section{CONCLUSION}

The solution of fast-storage based on C8051F340 and development process of USB communication are

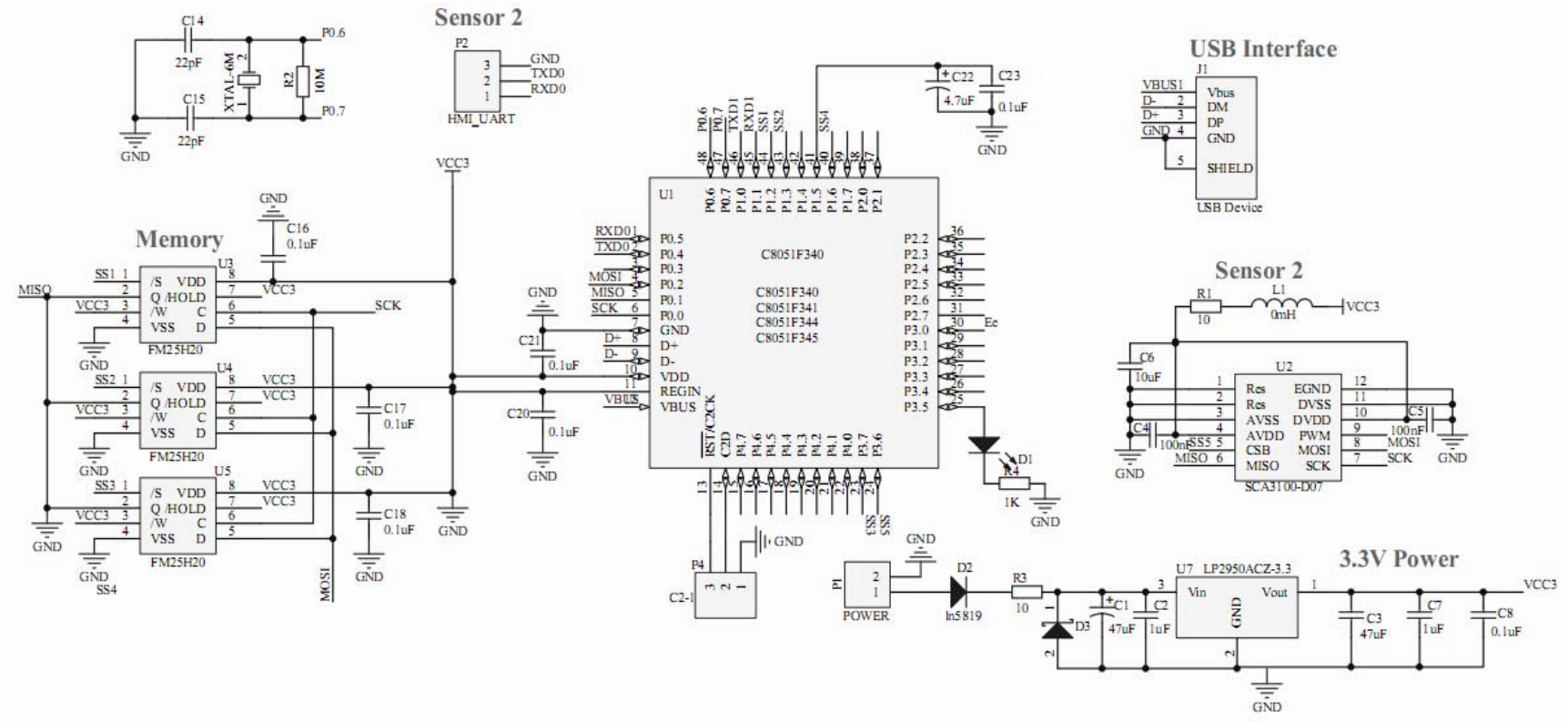

Figure 6. The circuit diagram of system of system constituent 